\title{
One-Dimensional Partially Asymmetric Simple Exclusion Process on a Ring with a Defect Particle
}

\author{
Tomohiro SASAMOTO * \\ Department of Physics, Graduate School of Science, \\ University of Tokyo, \\ Hongo 7-3-1, Bunkyo-ku, Tokyo 113-0033, Japan
}

\begin{abstract}
The effect of a moving defect particle for the one-dimensional partially asymmetric simple exclusion process on a ring is considered. The current of the ordinary particles, the speed of the defect particle and the density profile of the ordinary particles are calculated exactly. The phase diagram for the correlation length is identified. As a byproduct, the average and the variance of the particle density of the onedimensional partially asymmetric simple exclusion process with open boundaries are also computed.
\end{abstract}

\section{Introduction}

Recently, the one-dimensional asymmetric simple exclusion process (ASEP) has attracted much attention in various fields of science including mathematics, physics and biology [1 1 . There are several reasons for this. First of all, the ASEP has a lot of applications to realistic systems. For instance, the ASEP can be considered as a simplest model of traffic flow [5 7 . It is also used to study the dynamics of interface [8]. There are other applications as well. Second, the ASEP shows rich non-equilibrium behaviors such as the shock wave [9] 11, boundary induced phase transition [12], and the unusual dynamical scaling [13, 14]. Since there is no established framework for far-from equilibrium systems, the ASEP has played an important role in non-equilibrium statistical physics. Third, some properties of the ASEP can be studied exactly by the Bethe ansatz or the so-called matrix product ansatz. Since the effects of fluctuations become strong in low dimensions, mean-field analysis sometimes fails to give correct answers. Exact solutions give us an insight how to understand the problems which can not be solved exactly.

*e-mail: sasamoto@monet.phys.s.u-tokyo.ac.jp 
The ASEP is a one-dimensional lattice gas model. Each particle tends to hop to the right with rate $p$ and to the left with rate $q$. In addition, they interact with one another through hard-core exclusion interaction. In this article, we consider the stationary state of the ASEP on a ring with one defect particle. We sometimes refer to the particles as ordinary particles to distinguish them from the defect particle. We assume that the defect particle tends to hop to the right with rate $\alpha$ and that it can exchange its position with an ordinary particle on the left nearest neighboring site with rate $\beta$. By rescaling time, we can set $p=1$ without loss of generality. Then the above rules are represented as

$$
\begin{array}{lll}
10 \rightarrow 01 & \text { with rate } & 1 \\
01 \rightarrow 10 & \text { with rate } & q \\
20 \rightarrow 02 & \text { with rate } & \alpha \\
12 \rightarrow 21 & \text { with rate } & \beta
\end{array}
$$

Here 0,1 and 2 denote an empty site, an ordinary particle and the defect particle respectively. Several exact results have already been obtained for the $q=0$ case [15, 16]. This case is referred to as the "totally asymmetric" case in the following. The current of ordinary particles, the speed of the defect particle, the correlation length and the current fluctuation have been computed exactly for this special case. Much less is known for the "partially asymmetric" case where $q$ is not necessarily zero although some exact results were obtained in [17]. In this article, the model is studied for the $0<q<1$ case. We compute the current of the ordinary particles, the speed of the defect particle and the density profile of the ordinary particles seen from the defect particle. We exploit the connection of the present model to the partially ASEP with open boundaries, which was recently solved by using the theory of the $q$-orthogonal polynomials [18, 19]. We do not consider the $q \geq 1$ case, in which the current becomes zero in the thermodynamic limit. We remark that, for the partially ASEP with open boundaries, the $q=1$ case and the $q>1$ case were studied in 20] and 21] respectively.

The paper is organized as follows. In the next section, the stationary state of the process is constructed by the so-called matrix product ansatz. The current of the ordinary particles and the speed of the defect particle are calculated in section 3 . In section 4 , the average density profile is computed. The phase diagram for the correlation length is identified. The concluding remarks are given in the last section. In Appendix A, we compute the average and the variance of the particle density for the partially ASEP with open boundaries. This is a byproduct of the computations in the main text.

\section{Exact Stationary State in Matrix Product Form}

The stationary state of the process can be constructed by applying the so-called matrix product ansatz. This technique was first introduced in [22] to study directed animals. It was then applied to the ASEP in [23]. Since then this method has been successfully generalized and applied to many interesting phenomena. See for instance the references 
in [18. In the formalism of the matrix product ansatz, the stationary state of the process (1.1) is constructed as follows. Let us denote the number of particles as $N$ and the lattice length as $L+1$. To be specific we explain the construction mainly by using an example for the case $L=2, N=1$ : the case where there are one ordinary particle, one defect particle and one empty site on the chain with three sites. For this example, the stationary state can be written as a linear combination of the six configurations $012,021,102,120,201,210$ and is completely determined by specifying the corresponding six coefficients $P(012), P(021), P(102), P(120), P(201), P(210)$. Each coefficient $P\left(\tau_{1}, \tau_{2}, \tau_{3}\right)$ is the probability that the system is in a configuration $\tau_{1} \tau_{2} \tau_{2}$ with $\tau_{j}=0,1,2(j=1,2,3)$ for the stationary state.

Step 1: To each configuration $0,1,2$ at one site, we associate a matrix $E, D$ and $A$ respectively. The space on which these matrices act is not specified at this stage.

$$
\begin{aligned}
& 0 \leftrightarrow E \\
& 1 \leftrightarrow D \\
& 2 \leftrightarrow A
\end{aligned}
$$

Step 2: To each configuration $\tau_{1} \tau_{2} \tau_{3}$ of the whole lattice we associate a matrix product. For instance the configuration 120 is associated with the matrix product $D A E$.

Step 3: We construct a state, of which each coefficient is given by the trace of the matrix product. For the present example, the six coefficients $P^{(u)}(012), P^{(u)}(021), P^{(u)}(102)$, $P^{(u)}(120), P^{(u)}(201), P^{(u)}(210)$ are given by $\operatorname{Tr}(E D A), \operatorname{Tr}(E A D), \operatorname{Tr}(D E A), \operatorname{Tr}(D A E)$, $\operatorname{Tr}(A E D), \operatorname{Tr}(A D E)$ respectively. Here we denote the coefficients as $P^{(u)}\left(\tau_{1}, \tau_{2}, \tau_{3}\right)$ because the corresponding state is unnormalized. The coefficients of the normalized state $P\left(\tau_{1}, \tau_{2}, \tau_{3}\right)$ is obtained by simply dividing the unnormalized ones by the normalization constant,

$$
\begin{aligned}
Z_{L=3, N=1}= & \operatorname{Tr}(D E A)+\operatorname{Tr}(D A E)+\operatorname{Tr}(E D A) \\
& +\operatorname{Tr}(E A D)+\operatorname{Tr}(A D E)+\operatorname{Tr}(A E D) .
\end{aligned}
$$

In the above prescription, there is a problem about the existence of trace. We will see that we can take a special form of the matrix $A$ which ensures the existence of the trace.

Step 4: We can show that the constructed state is the stationary state of the process if the matrices $D, E, A$ satisfy the conditions,

$$
\begin{aligned}
D E-q E D & =\zeta(D+E), \\
\beta D A & =\zeta A, \\
\alpha A E & =\zeta A .
\end{aligned}
$$

Here $\zeta$ is an arbitrary number and is taken to be $\zeta=1-q$ hereafter. In addition, we set $\tilde{\alpha}=\alpha /(1-q), \tilde{\beta}=\beta /(1-q)$. The demonstration of the fact that the above algebraic 
relations are sufficient conditions for the stationarity of the process proceeds in almost the same manner as for the ASEP [23] and therefore is omitted. As we will see at the end of the section, we can take a matrix $A$ of the form $A=|V\rangle\langle W|$, where $|V\rangle$ and $\langle W|$ are some vectors. Then we notice that the above algebraic relations (2.3) are exactly the same as those for the partially ASEP with open boundaries [18, 23].

Step 5: Obviously, the construction of the stationary state above works for any choice of $L, N$. Explicitly, the coefficients $P\left(\tau_{1}, \tau_{2}, \ldots, \tau_{L}\right)$ of the normalized stationary state is given by

$$
P\left(\tau_{1}, \tau_{2}, \ldots, \tau_{L}\right)=\frac{1}{Z_{L, N}}\left\langle W\left|\prod_{j=1}^{L}\left[\tau_{j} D+\left(1-\tau_{j}\right) E\right]\right| V\right\rangle
$$

for the configuration $\tau_{1} \tau_{2} \ldots \tau_{L}$ which satisfies $\sum_{j=1}^{L} \tau_{j}=N$. Here the normalization constant $Z_{L, N}$ is

$$
Z_{L, N}=\sum_{\substack{\tau_{1}, \tau_{2}, \ldots, \tau_{L} \\ \sum_{j=1}^{L} \tau_{j}=N}}\left\langle W\left|\prod_{j=1}^{L}\left[\tau_{j} D+\left(1-\tau_{j}\right) E\right]\right| V\right\rangle
$$

The summation is over $\tau_{j}=0,1$ for $j=1,2, \ldots, L$ with the condition $\sum_{j=1}^{L} \tau_{j}=N$. Hence we have constructed the stationary state in matrix product form.

In this article, we will compute the current of the ordinary particles, the speed of the defect particle and the density profile of the ordinary particles seen from the defect particle. These quantities are written in terms of the matrices as follows.

The current of the ordinary particles is given by

$$
\begin{aligned}
J & =\operatorname{Prob}\left(\tau_{j}=1, \tau_{j+1}=0\right)-q \operatorname{Prob}\left(\tau_{j}=0, \tau_{j+1}=1\right)+\beta \operatorname{Prob}\left(\tau_{j}=1, \tau_{j+1}=2\right) \\
& =(1-q)\left[\frac{N}{L+1} \frac{Z_{L-1, N}}{Z_{L, N}}-\frac{L-N}{L+1} \frac{Z_{L-1, N-1}}{Z_{L, N}}\right] .
\end{aligned}
$$

The speed of the defect particle has a similar expression,

$$
\begin{aligned}
v & =\alpha \operatorname{Prob}\left(\tau_{j}=2, \tau_{j+1}=0\right)-\beta \operatorname{Prob}\left(\tau_{j}=1, \tau_{j+1}=2\right) \\
& =(1-q) \frac{Z_{L-1, N}-Z_{L-1, N-1}}{Z_{L, N}} .
\end{aligned}
$$

The average density of the ordinary particles at site $j$ seen from the defect particle reads

$$
\left\langle n_{j}\right\rangle_{L, N}^{(u)}=\sum_{\substack{\tau_{1}, \tau_{2}, \ldots, \hat{\tau}_{j}, \cdots, \tau_{L} \\ \tau_{1}+\cdots+\hat{\tau}_{j}+\cdots+\tau_{L}=N-1}}\left\langle W\left|\prod_{k=1}^{j-1}\left[\tau_{k} D+\left(1-\tau_{k}\right) E\right] D \prod_{k=j+1}^{L}\left[\tau_{k} D+\left(1-\tau_{k}\right) E\right]\right| V\right\rangle,
$$


where $\hat{\tau}_{j}$ indicates that it is omitted. This is an unnormalized quantity. The normalized density is given by $\left\langle n_{j}\right\rangle_{L, N}=\left\langle n_{j}\right\rangle_{L, N}^{(u)} / Z_{L, N}$.

Here we note that the process has a particle-hole symmetry which reduces our computations greatly. The process is invariant when the particles and holes are interchanged, the direction of particle hoppings are reversed, and $\alpha$ and $\beta$ are exchanged at the same time. In other words, the process is invariant under the change,

$$
\begin{gathered}
\text { particle } \leftrightarrow \text { hole } \\
\alpha \leftrightarrow \beta
\end{gathered}
$$

site number $j \leftrightarrow$ site number $L-j+1$.

Due to this symmetry, it is sufficient to obtain the density for the right half of the system, when we look at the system by setting the origin at the position of the defect particle. The density for the left half of the system is obtained by using the above symmetry as

$$
\left\langle n_{j}\right\rangle_{L, N}(\alpha, \beta)=1-\left\langle n_{L-j+1}\right\rangle_{L, L-N}(\beta, \alpha),
$$

where the dependence of $\left\langle n_{j}\right\rangle_{L}$ on the parameters $\alpha$ and $\beta$ are explicitly indicated.

So far, we have been considering the situation with a fixed number of particles. In other words, our discussions have been for the canonical ensemble. We are mainly interested in the physical quantities in the canonical ensemble. They can be directly compared with the results of the computer simulations because they are usually done for fixed particle numbers. However, it turns out that computations are much easier in the grand canonical ensemble. Since the meaning of chemical potential is unclear for our model, we define the grand canonical ensemble simply as a superposition of canonical ensembles. Let us introduce the fugacity $\xi^{2}$ which is associated with the ordinary particles. Then the coefficients $P_{\mathrm{GCE}}\left(\tau_{1}, \tau_{2}, \ldots, \tau_{L}\right)$ of the normalized stationary state for the grand canonical ensemble is compactly represented as

$$
P_{\mathrm{GCE}}\left(\tau_{1}, \tau_{2}, \ldots, \tau_{L}\right)=\frac{1}{Z_{L}(\xi)}\left\langle W\left|\prod_{j=1}^{L}\left[\tau_{j} \xi^{2} D+\left(1-\tau_{j}\right) E\right]\right| V\right\rangle .
$$

Here the normalization constant $Z_{L}(\xi)$ is a summation of the coefficients of the unnormalized states. In matrix notation, this is simply written as

$$
Z_{L}(\xi)=\left\langle W\left|C^{L}\right| V\right\rangle,
$$

with

$$
C=\xi^{2} D+E .
$$

We took the fugacity not as $\xi$ but as $\xi^{2}$. This is only for the notational simplicity in the formulae in the subsequent sections. But this causes an ambiguity; $\xi$ and $-\xi$ gives the same fugacity $\xi^{2}$. In the following we sometimes give explanation only for $\operatorname{Re} \xi>0$. But it 
is clear how the $\operatorname{Re} \xi<0$ case should be considered. One remark is in order. If we set $\xi=1$ in (2.11), $P_{\mathrm{GCE}}\left(\tau_{1}, \tau_{2}, \ldots, \tau_{L}\right)$ gives exactly the stationary state of the partially ASEP with open boundary condition in which there are particle input (resp. output) at the left (resp. right) end of the system with rate $\alpha$ (resp. $\beta$ ) [18, 23. In other words, the partially ASEP with open boundaries can be regarded as a superposition of the present one defect particle model with all numbers of particles $N=0,1,2, \ldots, L$ with equal weight. This fact allows us to compute the average and the variance of the density for the partially ASEP with open boundaries. This is done in the Appendix A.

Before closing the section, we give some notations, definitions and an example of the representation of algebraic relations (2.3). First we introduce $q$-shifted factorial,

$$
\begin{aligned}
& (a ; q)_{n}=(1-a)(1-a q)\left(1-a q^{2}\right) \cdots\left(1-a q^{n-1}\right), \\
& (a ; q)_{0}=1 .
\end{aligned}
$$

We also define

$$
(a ; q)_{\infty}=\prod_{j=0}^{\infty}\left(1-a q^{j}\right),
$$

for $|q|<1$. Since products of $q$-shifted factorials appear so often, we use the notations,

$$
\begin{aligned}
\left(a_{1}, a_{2}, \cdots, a_{k} ; q\right)_{\infty} & =\left(a_{1} ; q\right)_{\infty}\left(a_{2} ; q\right)_{\infty} \cdots\left(a_{k} ; q\right)_{\infty} \\
\left(a_{1}, a_{2}, \cdots, a_{k} ; q\right)_{n} & =\left(a_{1} ; q\right)_{n}\left(a_{2} ; q\right)_{n} \cdots\left(a_{k} ; q\right)_{n}
\end{aligned}
$$

An example of the representation of the algebra (2.3) is given by

$$
\begin{gathered}
D=\left[\begin{array}{ccccc}
1 & \sqrt{1-q} & 0 & 0 & \cdots \\
0 & 1 & \sqrt{1-q^{2}} & 0 & \\
0 & 0 & 1 & \sqrt{1-q^{3}} & \\
\vdots & \ddots & \ddots
\end{array}\right], \quad E=D^{T}, \quad A=|V\rangle\langle W|, \\
\left\langle W\left|=\kappa_{c}\left\langle a\left|=\kappa\left(1, \frac{a}{\sqrt{(q ; q)_{1}}}, \frac{a^{2}}{\sqrt{(q ; q)_{2}}}, \cdots\right), \quad\right| V\right\rangle=\kappa\right| b\right\rangle_{c}=\kappa\left(\begin{array}{c}
1 \\
\frac{b}{\sqrt{(q ; q)_{1}}} \\
\frac{b^{2}}{\sqrt{(q ; q)_{2}}} \\
\vdots
\end{array}\right),
\end{gathered}
$$

where the superscript $T$ indicates the transposition. Notice that the special form of the matrix $A$ ensures the existence of the trace. We introduced

$$
\begin{aligned}
& a=-1+\frac{1}{\tilde{\alpha}}=-1+\frac{1-q}{\alpha}, \\
& b=-1+\frac{1}{\tilde{\beta}}=-1+\frac{1-q}{\beta} .
\end{aligned}
$$


From now on, we restrict our attention to the case where $a>0$ and $b>0$, i.e., the case where $\alpha<1-q$ and $\beta<1-q$. This is mainly for the simplicity of the discussions. We do not expect there appears another phase transition if we consider $a<0$ or $b<0$ case. The constant $\kappa$ in $(2.18 \mathrm{~b})$ is takes as $\kappa^{2}=(a b ; q)_{\infty}$ so that $\langle W \mid V\rangle=1$. An important fact about these matrices is that they are related to the so-called $q$-orthogonal polynomials [24-26]. It is this connection that allows us to compute the physical quantities exactly in the subsequent sections. The relationship between the matrix product ansatz for the partially ASEP and the theory of the $q$-orthogonal polynomials was first clarified in [18 and has been exploited to study the partially ASEP with open boundaries in [19,21.

\section{The Current}

In this section, we compute the current of the ordinary particles (2.6) and the speed of the defect particle (2.7) in the thermodynamic limit. We need to know the asymptotic expression of $Z_{L, N}$ when $L, N \rightarrow \infty$ with $\rho=N / L$ fixed.

\subsection{Exact Formula for $Z_{L}(\xi)$}

First we give the exact expression of $Z_{L}(\xi)$ in the form of contour integral. Since the derivation is almost the same as that in [18], the proof is omitted. The result is

$$
Z_{L}(\xi)=\frac{(q, a b ; q)_{\infty}}{4 \pi i} \int_{C} d z \frac{\left(z^{2}, z^{-2} ; q\right)_{\infty}\left[(1+\xi z)\left(1+\xi z^{-1}\right)\right]^{L}}{\left(a \xi^{-1} z, a \xi^{-1} z^{-1}, b \xi z, b \xi z^{-1} ; q\right)_{\infty}}
$$

The contour $C$ of the integral above is such that it includes all poles of the type $a \xi^{-1} q^{k}$ and $b \xi q^{k}$ with $k=0,1,2, \cdots$ whilest it excludes all poles of the type $a^{-1} \xi q^{-k}$ and $b^{-1} \xi^{-1} q^{-k}$ with $k=0,1,2, \cdots$. When $a \xi^{-1}<1$ and $b \xi<1$ hold, the contour is simply a unit circle and $Z_{L}(\xi)$ reduces to the single integral on the real line. But when $a \xi^{-1}>1$ or $b \xi>1$ there appear other terms as well. In general, when

$$
\begin{gathered}
a \xi^{-1}>a \xi^{-1} q>\cdots>a \xi^{-1} q^{n^{(a)}}>1>a \xi^{-1} q^{n^{(a)}+1}>\cdots \\
b \xi>b \xi q>\cdots>b \xi q^{n^{(b)}}>1>b \xi q^{n^{(b)}+1}>\cdots
\end{gathered}
$$

where $n^{(a)}$ and $n^{(b)}$ are some non-negative integers, we have

$$
Z_{L}(\xi)=Z_{L}^{(0)}(\xi)+Z_{L}^{(a)}(\xi)+Z_{L}^{(b)}(\xi)
$$


Explicit expressions for the above three terms read

$$
\begin{aligned}
& Z_{L}^{(0)}(\xi)=\frac{(q, a b ; q)_{\infty}}{2 \pi} \int_{0}^{\pi} d \theta \frac{\left(e^{2 i \theta}, e^{-2 i \theta} ; q\right)_{\infty}\left[\left(1+\xi e^{i \theta}\right)\left(1+\xi e^{-i \theta}\right)\right]^{L}}{\left(a \xi^{-1} e^{i \theta}, a \xi^{-1} e^{-i \theta}, b \xi e^{i \theta}, b \xi e^{-i \theta} ; q\right)_{\infty}} \\
& Z_{L}^{(a)}(\xi)=(q, a b ; q)_{\infty} \sum_{j=0}^{n^{(a)}} w_{j}^{(a)}\left[\left(1+a q^{j}\right)\left(1+\xi^{2} a^{-1} q^{-j}\right)\right]^{L} \\
& Z_{L}^{(b)}(\xi)=(q, a b ; q)_{\infty} \sum_{j=0}^{n^{(b)}} w_{j}^{(b)}\left[\left(1+\xi^{2} b q^{j}\right)\left(1+b^{-1} q^{-j}\right)\right]^{L},
\end{aligned}
$$

with

$$
\begin{aligned}
w_{j}^{(a)} & =\frac{\left(a^{-2} \xi^{2} ; q\right)_{\infty}\left(a^{2} \xi^{-2}, a b ; q\right)_{j}\left(1-\xi^{-2} a^{2} q^{2 j}\right) \xi^{2 j}}{\left(q, a b, a^{-1} b \xi^{2} ; q\right)_{\infty}\left(q, a b^{-1} \xi^{-2} q ; q\right)_{j}\left(1-\xi^{-2} a^{2}\right) q^{j^{2}} a^{3 j} b^{j}}, \\
w_{j}^{(b)} & =\frac{\left(b^{-2} \xi^{-2} ; q\right)_{\infty}\left(b^{2} \xi^{2}, a b ; q\right)_{j}\left(1-\xi^{2} b^{2} q^{2 j}\right)}{\left(q, a b, a b^{-1} \xi^{-2} ; q\right)_{\infty}\left(q, a^{-1} b \xi^{2} q ; q\right)_{j}\left(1-\xi^{2} b^{2}\right) q^{j^{2}} a^{j} b^{3 j} \xi^{2 j}} .
\end{aligned}
$$

Off course when $a \xi^{-1}<1$ and/or $b \xi<1$, we should omit $Z_{L}^{(a)}$ and/or $Z_{L}^{(b)}$ in (3.3).

The asymptotic behavior of $Z_{L}^{(0)}(\xi)$ can be evaluated by applying the steepest descent method, whereas the asymptotic behaviors of $Z_{L}^{(a)}(\xi)$ and $Z_{L}^{(b)}(\xi)$ are simply given by the $j=0$ terms in the summation. We find

$$
\begin{gathered}
Z_{L}^{(0)}(\xi) \simeq \frac{(q, a b ; q)_{\infty}\left[(1+\xi)\left(1+\xi^{-1}\right)\right]^{3 / 2}}{2 \sqrt{\pi}\left(a \xi^{-1}, b \xi ; q\right)_{\infty}^{2} L^{3 / 2}}(1+\xi)^{2 L} \\
Z_{L}^{(a)}(\xi) \simeq \frac{\left(\xi^{2} a^{-2} ; q\right)_{\infty}}{\left(a^{-1} b \xi^{2} ; q\right)_{\infty}}\left[(1+a)\left(1+\xi^{2} a^{-1}\right)\right]^{L} \\
Z_{L}^{(b)}(\xi) \simeq \frac{\left(\xi^{-2} b^{-2} ; q\right)_{\infty}}{\left(a b^{-1} \xi^{-2} ; q\right)_{\infty}}\left[\left(1+\xi^{2} b\right)\left(1+b^{-1}\right)\right]^{L}
\end{gathered}
$$

as $L \rightarrow \infty$ when $\operatorname{Re} \xi>0$.

\subsection{Asymptotic Formula for $Z_{L, N}$}

In this subsection we study the asymptotic behavior of $Z_{L, N}$. First we notice

$$
Z_{L}(\xi)=\sum_{N=0}^{L} Z_{L, N} \xi^{2 N}
$$

The average density of particles is given by

$$
\langle\rho\rangle=\xi^{2} \lim _{L \rightarrow \infty} \frac{1}{L} \frac{\partial}{\partial \xi^{2}} \ln Z_{L}(\xi) .
$$


Basically we can expect that each value of the fugacity $\xi^{2}$ corresponds to each value of the density. Then the density-fugacity relation is invertible and the equivalence of the canonical and the grand canonical ensemble holds. For our model, however, there appears a situation where the equivalence of the ensembles fails. For this case, we have to go back to (3.12) and use the inversion of it,

$$
Z_{L, N}=\frac{1}{2 \pi i} \int_{C_{1}} d \xi \frac{Z_{L}(\xi)}{\xi^{2 N+1}}
$$

where $C_{1}$ is a contour which encircles the origin anti-clockwise. Notice that (3.14) is always true for a finite $L$ since $Z_{L}(\xi)$ is a polynomial of degree $2 N$. This formula also has an advantage that it allows us to obtain not only the exponent of the asymptotic behavior of $Z_{L, N}$ but also the prefactor of it.

In order to find the relationship between the density and the fugacity and derive the asymptotic expression of $Z_{L, N}$, we need to know, for a give value of $\xi$, which term in (3.3) gives the main contribution to $Z_{L}(\xi)$. For notational convenience we define $\xi_{0}=\rho /(1-\rho)$.

When $\tilde{\alpha}+\tilde{\beta}>1 \quad(a b<1)$

- Case $0<\xi<a$

The main contribution to $Z_{L}(\xi)$ comes from $Z_{L}^{(a)}$. The density-fugacity relation (3.13) gives $\xi=\sqrt{a \xi_{0}}\left(\equiv \xi_{a}\right)$. In order for this value to be in the region $0<\xi<a$, the density $\rho$ should satisfy $\rho<1-\tilde{\alpha}<\tilde{\beta}$. The asymptotic behavior of $Z_{L, N}^{(a)}$ can be obtained by applying the steepest descent method.

- Case $a<\xi<b^{-1}$

The main contribution to $Z_{L}(\xi)$ comes from $Z_{L}^{(0)}$. The density-fugacity relation (3.13) gives $\xi=\xi_{0}$. In order for this value to be in the region $a<\xi<b^{-1}$, the density $\rho$ should satisfy $1-\tilde{\alpha}<\rho<\tilde{\beta}$. The asymptotic behavior of $Z_{L, N}^{(b)}$ can be obtained by applying the steepest descent method.

- Case $\xi>b^{-1}$

The main contribution to $Z_{L}(\xi)$ comes from $Z_{L}^{(b)}$. The density-fugacity relation (3.13) gives $\xi=\sqrt{\xi_{0} / b}\left(\equiv \xi_{b}\right)$. In order for this value to be in the region $\xi>b^{-1}$, the density $\rho$ should satisfy $1-\tilde{\alpha}<\tilde{\beta}<\rho$. The asymptotic behavior of $Z_{L, N}^{(0)}$ can be obtained by applying the steepest descent method.

When $\tilde{\alpha}+\tilde{\beta}<1 \quad(a b>1)$

- Case $0<\xi<\sqrt{a / b}$

The main contribution to $Z_{L}(\xi)$ comes from $Z_{L}^{(a)}$. The density-fugacity relation (3.13) gives $\xi=\xi_{a}$. In order for this value to be in the region $0<\xi<\sqrt{a / b}$, the density $\rho$ should satisfy $\rho<\tilde{\beta}<1-\tilde{\alpha}$. 
- Case $\xi>\sqrt{a / b}$

The main contribution to $Z_{L}(\xi)$ comes from $Z_{L}^{(b)}$. The density-fugacity relation (3.13) gives $\xi=\xi_{b}$. In order for this value to be in the region $\xi>\sqrt{a / b}$, the density $\rho$ should satisfy $\tilde{\beta}<1-\tilde{\alpha}<\rho$.

- Case $\xi=\sqrt{a / b}$

So far we have not seen the values of the fugacity which corresponds to the region $\tilde{\beta}<\rho<1-\tilde{\alpha}$. It turns out that this is related to the fact that the density-fugacity relation (3.13) breaks down at $\xi=\sqrt{a / b}$. Notice that $Z_{L, N}^{(a)}(\xi)$ and $Z_{L, N}^{(b)}(\xi)$ in (3.10) and (3.11) have a pole at this point. Physically, this is related to the existence of a shock. It might seem difficult to know the asymptotic behavior of $Z_{L, N}$ for this case. But this is not the case. Let us employ (3.14) and take the contour as a circle $C_{R}$ with the radius $R$. As long as $L$ is finite, we can change the radius $R$ as we wish. To know the asymptotic behavior of $Z_{L, N}$, we want to use (3.9)-(3.11) and apply the steepest descent method. It turns out that the saddle point associated with $Z_{L}^{(a)}(\xi)$ (resp. $Z_{L}^{(b)}(\xi)$ ) is at $\xi=\xi_{a}$ (resp. $\xi=\xi_{b}$ ) and the contribution from $Z_{L}^{(0)}(\xi)$ is smaller than the others. Hence, to apply the steepest descent method, we have to take the radius of the contour as $R_{1}=\xi_{a}(>\sqrt{a / b})$ for $Z_{L}^{(a)}(\xi)$ and as $R_{2}=\xi_{b}(<\sqrt{a / b})$ for $Z_{L}^{(b)}(\xi)$. At first let us take $R$ as $R_{2}$. We have

$$
\begin{aligned}
Z_{L, N} \simeq & \frac{1}{2 \pi i} \int_{C_{R_{2}}} \frac{d \xi}{\xi} \frac{\left(a^{-2} \xi^{2} ; q\right)_{\infty}}{\left(a^{-1} b \xi^{2} ; q\right)_{\infty}} \frac{\left[(1+a)\left(1+\xi^{2} a^{-1}\right)\right]^{L}}{\xi^{2 N}} \\
& +\frac{1}{2 \pi i} \int_{C_{R_{2}}} \frac{d \xi}{\xi} \frac{\left(b^{-2} \xi^{-2} ; q\right)_{\infty}}{\left(a b^{-1} \xi^{-2} ; q\right)_{\infty}} \frac{\left[\left(1+\xi^{2} b\right)\left(1+b^{-1}\right)\right]^{L}}{\xi^{2 N}} .
\end{aligned}
$$

To apply the steepest descent method to the first term, we have to modify $C_{R_{2}}$ to $C_{R_{1}}$. Since the integrand of the first term has a pole at $\sqrt{a / b}$, there appears a contribution from the pole when the contour is modified. More explicitly, we see

$$
\begin{aligned}
& \frac{1}{2 \pi i} \int_{C_{R_{1}}} \frac{d \xi}{\xi} \frac{\left(a^{-2} \xi^{2} ; q\right)_{\infty}}{\left(a^{-1} b \xi^{2} ; q\right)_{\infty}} \frac{\left[(1+a)\left(1+\xi^{2} a^{-1}\right)\right]^{L}}{\xi^{2 N}} \\
= & \frac{1}{2 \pi i} \int_{C_{R_{2}}} \frac{d \xi}{\xi} \frac{\left(a^{-2} \xi^{2} ; q\right)_{\infty}}{\left(a^{-1} b \xi^{2} ; q\right)_{\infty}} \frac{\left[(1+a)\left(1+\xi^{2} a^{-1}\right)\right]^{L}}{\xi^{2 N}}-\frac{\left(a^{-1} b^{-1} ; q\right)_{\infty}(1+a)^{L}\left(1+b^{-1}\right)^{L}}{(q ; q)_{\infty}\left(a b^{-1}\right)^{N}} .
\end{aligned}
$$

Now we can apply the steepest descent method to the two integrals. But it turns out that the contribution from the pole gives the asymptotic behavior of $Z_{L, N}$ for this case.

Combining the above results, we see that there are four regions in the $\tilde{\alpha}-\tilde{\beta}$ plane, in each of which the asymptotic behavior of $Z_{L, N}$ in the thermodynamic limit has a different form. We refer to these four phases as Phase $\mathbf{A}, \mathbf{B}, \mathbf{C}$ and $\mathbf{D}$. The asymptotic expression of $Z_{L, N}$ in each phase is given by 
- Phase A: $\tilde{\alpha}<1-\rho, \tilde{\beta}>\rho$

$$
Z_{L, N} \simeq Z_{L, N}^{(a)} \simeq \frac{\left(a^{-1} \xi_{0} ; q\right)_{\infty}}{\left(b \xi_{0} ; q\right)_{\infty}} \frac{1}{\sqrt{2 \pi} \tilde{\alpha}^{L-N}(1-\tilde{\alpha})^{N} \rho^{N+1 / 2}(1-\rho)^{L-N+1 / 2}} .
$$

- Phase B: $\tilde{\alpha}>1-\rho, \tilde{\beta}<\rho$

$$
Z_{L, N} \simeq Z_{L, N}^{(b)} \simeq \frac{\left(b^{-1} \xi_{0}^{-1} ; q\right)_{\infty}}{\left(a \xi_{0}^{-1} ; q\right)_{\infty}} \frac{1}{\sqrt{2 \pi} \tilde{\beta}^{N}(1-\tilde{\beta})^{L-N} \rho^{N+1 / 2}(1-\rho)^{L-N+1 / 2}} .
$$

- Phase C: $\tilde{\alpha}>1-\rho, \tilde{\beta}>\rho$

$$
Z_{L, N} \simeq Z_{L, N}^{(0)} \simeq \frac{\tilde{\alpha} \tilde{\beta}(\tilde{\alpha}+\tilde{\beta}+1)(q, a b q ; q)_{\infty}}{(\tilde{\alpha}+\rho-1)^{2}(\tilde{\beta}-\rho)^{2}\left(\xi_{0}^{-1} a q, \xi_{0} b q ; q\right)_{\infty}^{2}} \frac{1}{2 \pi L^{2} \rho^{2 N}(1-\rho)^{2 L-2 N}}
$$

- Phase D: $\tilde{\alpha}<1-\rho, \tilde{\beta}<\rho$

$$
Z_{L, N} \simeq \frac{(1-\tilde{\alpha}-\tilde{\beta})\left(a^{-1} b^{-1} q ; q\right)_{\infty}}{(1-\tilde{\alpha})(1-\tilde{\beta})(q ; q)_{\infty}} \frac{1}{\tilde{\alpha}^{L-N}(1-\tilde{\alpha})^{N} \tilde{\beta}^{N}(1-\tilde{\beta})^{L-N}}\left(=Z_{L, N}^{(d)}\right) .
$$

These are simple generalization of the results in [15] for the totally asymmetric case.

\section{3 $J$ and $v$ in the Thermodynamic Limit}

Now it is straight forward to calculate the current of the ordinary particles $J$ and the speed of the defect particle $v$ in the thermodynamic limit. The results are summarized as follows:

- Phase A: $J=(1-q) \rho(1-\rho)$ and $v=(1-q)(\tilde{\alpha}-\rho)$.

- Phase B: $J=(1-q) \rho(1-\rho)$ and $v=(1-q)(1-\tilde{\beta}-\rho)$.

- Phase C: $J=(1-q) \rho(1-\rho)$ and $v=(1-q)(1-2 \rho)$.

- Phase $\mathbf{D}: J=(1-q)[\rho(\tilde{\alpha}-\tilde{\beta})+\tilde{\beta}(1-\tilde{\alpha})]$ and $v=(1-q)(\tilde{\alpha}-\tilde{\beta})$.

\section{Density Profile}

Now we turn to consider the average density profile. As in the previous section, we first calculate the density in the grand canonical ensemble. In the grand canonical ensemble, the (unnormalized) average density at site $j$ is defined by

$$
\left\langle n_{j}\right\rangle_{L}^{(u)}(\xi)=\sum_{\tau_{1}, \tau_{2}, \ldots, \hat{\tau}_{j}, \cdots, \tau_{L}}\left\langle V\left|\prod_{k=1}^{j-1}\left[\tau_{j} \xi^{2} D+\left(1-\tau_{j}\right) E\right] \xi^{2} D \prod_{k=j+1}^{L}\left[\tau_{j} \xi^{2} D+\left(1-\tau_{j}\right) E\right]\right| V\right\rangle .
$$


To translate the results in the grand canonical ensemble into those in the canonical ensemble, we need the formula,

$$
\left\langle n_{j}\right\rangle_{L, N}^{(u)}=\frac{1}{2 \pi i} \int_{C_{1}} d \xi \frac{\left\langle n_{j}\right\rangle_{L}^{(u)}(\xi)}{\xi^{2 N+1}}
$$

Now we explain the main idea how to compute $\left\langle n_{j}\right\rangle_{L}^{(u)}(\xi)$. Details of the computation are not presented here since they are similar to those in [19]. Since it is easier to calculate the density difference than the density itself, we rewrite the density at site $j$ as

$$
\left\langle n_{j}\right\rangle_{L}=\sum_{k=j}^{L-1}\left(\left\langle n_{k}\right\rangle_{L}^{(u)}(\xi)-\left\langle n_{k+1}\right\rangle_{L}^{(u)}(\xi)\right)+\left\langle n_{L}\right\rangle_{L}^{(u)}(\xi)
$$

At the right boundary, we have

$$
\left\langle n_{L}\right\rangle_{L}^{(u)}(\xi)=\frac{\xi^{2}}{\tilde{\beta}}\left\langle W\left|C^{L-1}\right| V\right\rangle .
$$

Similar formula can be found for the left boundary as well. As for the computation of $\left\langle n_{k}\right\rangle_{L}^{(u)}(\xi)-\left\langle n_{k+1}\right\rangle_{L}^{(u)}(\xi)$, we notice that

$$
\begin{aligned}
\left\langle n_{k}\right\rangle_{L}^{(u)}(\xi)-\left\langle n_{k+1}\right\rangle_{L}^{(u)}(\xi) & =\xi^{2}\left(\left\langle W\left|C^{k-1} D C^{L-k}\right| V\right\rangle-\left\langle W\left|C^{k} D C^{L-k-1}\right| V\right\rangle\right) \\
& =\xi^{2}\left\langle W\left|C^{k-1}(D C-C D) C^{L-k-1}\right| V\right\rangle \\
& =\xi^{2}\left\langle W\left|C^{k-1}(D E-E D) C^{L-k-1}\right| V\right\rangle .
\end{aligned}
$$

By using the two facts that $D E-E D$ is a simple diagonal matrix and that the resultant series can be summed up by using a formula for the $q$-Hermite polynomials, it turns out to be possible to derive an integral expression of $\left\langle n_{k}\right\rangle_{L}^{(u)}(\xi)-\left\langle n_{k+1}\right\rangle_{L}^{(u)}(\xi)$. If we sum up these terms from $k=j$ to $k=L-1$, after some computation, we get

$$
\sum_{k=j}^{L-1}\left(\left\langle n_{k}\right\rangle_{L}^{(u)}(\xi)-\left\langle n_{k+1}\right\rangle_{L}^{(u)}(\xi)\right)=I_{1}+I_{2},
$$

where

$$
\begin{aligned}
I_{1}= & \frac{\xi}{4}(a b ; q)_{\infty}(q ; q)_{\infty}^{3} \int \frac{d z_{1}}{2 \pi i z_{1}} \int \frac{d z_{2}}{2 \pi i z_{2}} \\
& \times \frac{\left(z_{1}^{2}, z_{1}^{-2}, z_{2}^{2}, z_{2}^{-2} ; q\right)_{\infty}\left[\left(1+\xi z_{1}\right)\left(1+\xi z_{1}^{-1}\right)\right]^{L-1}}{\left(a \xi^{-1} z_{1}, a \xi^{-1} z_{1}^{-1}, q z_{1} z_{2}, q z_{1}^{-1} z_{2}^{-1}, q z_{1} z_{2}^{-1}, q z_{1}^{-1} z_{2}^{-1}, b \xi z_{2}, b \xi z_{2}^{-1} ; q\right)_{\infty}\left(z_{2}+z_{2}^{-1}-z_{1}-z_{1}^{-1}\right)}, \\
I_{2}= & \frac{\xi}{4}(a b ; q)_{\infty}(q ; q)_{\infty}^{3} \int \frac{d z_{1}}{2 \pi i z_{1}} \int \frac{d z_{2}}{2 \pi i z_{2}} \\
& \times \frac{\left(z_{1}^{2}, z_{1}^{-2}, z_{2}^{2}, z_{2}^{-2} ; q\right)_{\infty}\left[\left(1+\xi z_{1}\right)\left(1+\xi z_{1}^{-1}\right)\right]^{j-1}\left[\left(1+\xi z_{2}\right)\left(1+\xi z_{2}^{-1}\right)\right]^{L-j}}{\left(a \xi^{-1} z_{1}, a \xi^{-1} z_{1}^{-1}, q z_{1} z_{2}, q z_{1}^{-1} z_{2}^{-1}, q z_{1} z_{2}^{-1}, q z_{1}^{-1} z_{2}^{-1}, b \xi z_{2}, b \xi z_{2}^{-1} ; q\right)_{\infty}\left(z_{2}+z_{2}^{-1}-z_{1}-z_{1}^{-1}\right)} .
\end{aligned}
$$


When $0<a \xi^{-1}, b \xi<1$, the contours of $z_{1}$ and $z_{2}$ are both unit circles. For other values of the parameters, the contours are modified so that the analyticity of $I_{1}$ and $I_{2}$ are ensured.

Combining the results for $\left\langle n_{j}\right\rangle_{L}^{(u)}(\xi)$ with the formula (4.2), we obtain the density profile of the ordinary particles in the canonical ensemble. The density at the right boundary is computed by using (4.4). The integral $I_{1}$ gives us the density at bulk region, which turns out to be $\rho$ except for the phase $\mathbf{D}$. Off course this is consistent with the fact that we are now dealing with the system with the fixed density $\rho$. The integral $I_{2}$ contains the information about how the density decays near the right boundary. The density near the left boundary can be found by noticing the symmetry (2.10). The results are summarized in the following.

Phase $\mathbf{A}\left(\tilde{\alpha}<1-\rho\right.$ and $\tilde{\beta}>\rho ; a>\xi_{0}$ and $\left.b<1 / \xi_{0}\right)$

The average density in the bulk region takes the constant value $\rho$. The densities at boundaries are given by

$$
\left\langle n_{1}\right\rangle_{L}=\rho, \quad\left\langle n_{L}\right\rangle_{L}=\frac{1-\tilde{\alpha}}{\tilde{\beta}} \rho
$$

Notice that the density near the left boundary does not change and takes the constant value $\rho$. Computation of the density near the right boundary shows that the phase $\mathbf{A}$ subdivides into three phases.

- Phase $\mathbf{A}_{1}$

This phase corresponds to

$$
\rho<\tilde{\beta}<\frac{\rho}{\rho+(1-\rho) q}, \quad \tilde{\beta}<\frac{\sqrt{(1-\tilde{\alpha}) \rho}}{\sqrt{\tilde{\alpha}(1-\rho)}+\sqrt{(1-\tilde{\alpha}) \rho}}
$$

in the $\tilde{\alpha}-\tilde{\beta}$ plane and to

$$
q / \xi_{0}<b<1 / \xi_{0}, \quad b>1 / \sqrt{a \xi_{0}}
$$

in the $a-b$ plane. Let us denote the distance from the right boundary as $l=L-j+1$ from now on. Then the density near the right boundary decays exponentially as

$$
\left\langle n_{j}\right\rangle_{L}=\rho-\frac{\left(a b^{2} \xi_{0}-1\right)\left(q, a^{-1} b^{-2} \xi_{0}^{-1} q ; q\right)_{\infty}}{\left(1+a b \xi_{0}\right)(1+b)\left(a^{-1} b^{-1} q, b^{-1} \xi_{0}^{-1} q ; q\right)_{\infty}} \exp [-l / r]
$$

with the correlation length,

$$
r^{-1}=-\ln \left[\frac{\tilde{\alpha}(1-\rho)}{1-\tilde{\beta}}+\frac{(1-\tilde{\alpha}) \rho}{\tilde{\beta}}\right] .
$$


- Phase $\mathbf{A}_{2}$

This phase corresponds to

$$
\frac{(1-\rho) q^{2}}{\rho+(1-\rho) q^{2}}<\tilde{\alpha}<1-\rho, \quad \tilde{\beta}>\frac{\sqrt{(1-\tilde{\alpha}) \rho}}{\sqrt{\tilde{\alpha}(1-\rho)}+\sqrt{(1-\tilde{\alpha}) \rho}}
$$

in the $\tilde{\alpha}-\tilde{\beta}$ plane and to

$$
\xi_{0}<a<\xi_{0} q^{-2}, \quad b<1 / \sqrt{a \xi_{0}}
$$

in the $a-b$ plane. The density profile near the right boundary decays exponentially as

$$
\left\langle n_{j}\right\rangle_{L}=\rho-\frac{\sqrt{\xi_{0}}\left(1+a \xi_{0}\right)\left(a b, b \xi_{0} ; q\right)_{\infty}(q ; q)_{\infty}^{4}}{2 \sqrt{a \pi}\left(\sqrt{a \xi_{0}^{-1}} q, \sqrt{a^{-1} \xi_{0}}, b \sqrt{a \xi_{0}} ; q\right)_{\infty}^{2}} \frac{\exp [-l / r]}{l^{\frac{3}{2}}},
$$

with the correlation length,

$$
r^{-1}=-2 \ln [\sqrt{\tilde{\alpha}(1-\rho)}+\sqrt{(1-\tilde{\alpha}) \rho}] .
$$

The decay of the density is not purely exponential but with algebraic corrections.

- Phase $\mathbf{A}_{3}$

This phase corresponds to

$$
\tilde{\alpha}<\frac{(1-\rho) q^{2}}{\rho+(1-\rho) q^{2}}, \quad \tilde{\beta}>\frac{\rho}{\rho+(1-\rho) q}
$$

in the $\tilde{\alpha}-\tilde{\beta}$ plane and to

$$
a>\xi_{0} q^{-2}, \quad b<q \xi_{0}^{-1}
$$

in the $a-b$ plane. The density profile near the right boundary decays exponentially as

$$
\left\langle n_{j}\right\rangle_{L}=\rho-\frac{\xi_{0}(1-a b)\left(1-a^{-1} \xi_{0} q^{-2}\right)}{\left(1-b \xi_{0} q^{-1}\right)(1+a q)\left(1+\xi_{0} q^{-1}\right)} \exp [-l / r]
$$

with the correlation length,

$$
r^{-1}=\ln \frac{q}{[\tilde{\alpha}+(1-\tilde{\alpha}) q][\rho+(1-\rho) q]} .
$$

Phase B $\left(\tilde{\alpha}>1-\rho\right.$ and $\tilde{\beta}<\rho ; a<\xi_{0}$ and $\left.b>1 / \xi_{0}\right)$

This phase is symmetric to the Phase $\mathbf{A}$. All results can be obtained by using the symmetry (2.9). The average density in the bulk region takes the constant value $\rho$ and does not change near the right boundary. The densities at boundaries are given by

$$
\left\langle n_{1}\right\rangle_{L}=1-\frac{1-\tilde{\beta}}{\tilde{\alpha}}(1-\rho), \quad\left\langle n_{L}\right\rangle_{L}=\rho .
$$




\section{- Phase $\mathbf{B}_{1}$}

This phase corresponds to

$$
1-\rho<\tilde{\alpha}<\frac{1-\rho}{1-\rho+\rho q}, \quad \tilde{\alpha}<\frac{\sqrt{(1-\tilde{\beta})(1-\rho)}}{\sqrt{\tilde{\beta} \rho}+\sqrt{(1-\tilde{\beta})(1-\rho)}}
$$

in the $\tilde{\alpha}-\tilde{\beta}$ plane and to

$$
\xi_{0} q<a<\xi_{0}, \quad b>\frac{\xi_{0}}{a^{2}}
$$

in the $a-b$ plane. The density near the left boundary decays exponentially with the correlation length,

$$
r^{-1}=-\ln \left[\frac{\tilde{\beta} \rho}{1-\tilde{\alpha}}+\frac{(1-\tilde{\beta})(1-\rho)}{\tilde{\alpha}}\right] .
$$

- Phase $\mathbf{B}_{2}$

This phase corresponds to

$$
\frac{\rho q^{2}}{1-\rho+\rho q^{2}}<\tilde{\alpha}<1-\rho, \quad \tilde{\alpha}>\frac{\sqrt{(1-\tilde{\beta})(1-\rho)}}{\sqrt{\tilde{\beta} \rho}+\sqrt{(1-\tilde{\beta})(1-\rho)}}
$$

in the $\tilde{\alpha}-\tilde{\beta}$ plane and to

$$
\xi_{0}^{-1}<b<\xi_{0}^{-1} q^{-2}, \quad a<b<\frac{\xi_{0}}{a^{2}}
$$

in the $a-b$ plane. The density profile near the left boundary decays exponentially with the correlation length,

$$
r^{-1}=-2 \ln [\sqrt{\tilde{\beta} \rho}+\sqrt{(1-\tilde{\beta})(1-\rho)}] .
$$

- Phase $\mathbf{B}_{3}$

This phase corresponds to

$$
\tilde{\beta}<\frac{\rho q^{2}}{1-\rho+\rho q^{2}}, \quad \tilde{\alpha}>\frac{1-\rho}{1-\rho+\rho q}
$$

in the $\tilde{\alpha}-\tilde{\beta}$ plane and to

$$
b>\xi_{0}^{-1} q^{-2}, \quad a<\xi_{0} q
$$


in the $a-b$ plane. The density profile near the left boundary decays exponentially with the correlation length,

$$
r^{-1}=\ln \frac{q}{[\tilde{\beta}+(1-\tilde{\beta}) q][1-\rho+\rho q]} .
$$

Phase $\mathbf{C}\left(1-\tilde{\alpha}<\rho<\tilde{\beta} ; a<\xi_{0}\right.$ and $\left.b<\xi_{0}^{-1}\right)$

The average density in the bulk region takes the constant value $\rho$. The densities at boundaries are given by

$$
\left\langle n_{1}\right\rangle_{L}=1-\frac{1}{\tilde{\alpha}}(1-\rho)^{2}, \quad\left\langle n_{L}\right\rangle_{L}=\frac{\rho^{2}}{\tilde{\beta}} .
$$

The average density decays near the right boundary as

$$
\left\langle n_{j}\right\rangle_{L}=\rho-\sqrt{\frac{\rho(1-\rho)}{\pi}} \frac{1}{l^{\frac{1}{2}}} .
$$

The density decays algebraically and hence the correlation length is infinite. The density decay at the left boundary can be obtained from the symmetry relation (2.10). This is the same as the result for the $q=0$ case in [15].

Phase D $\left(\tilde{\alpha}<1-\rho\right.$ and $\tilde{\beta}<\rho ; a>\xi_{0}$ and $\left.b>\xi_{0}^{-1}\right)$

In this phase, there is a shock. Hence the average density at bulk region is not a constant. For the densities at the boundaries, we have

$$
\left\langle n_{1}\right\rangle_{L}=\tilde{\beta}, \quad\left\langle n_{L}\right\rangle_{L}=1-\tilde{\alpha} .
$$

There are two regions in the system. One is the low density region with the density $\tilde{\beta}$ at left side and the other is the high density region with the density $1-\tilde{\alpha}$ at right side. The two regions are separated by a sharp interface, which we call a shock. When $L$ is large, the average density profile is described by a continuous function $n(x)$ in terms of the rescaled variable $x=j / L(0 \leq x \leq 1)$. The derivative of $n(x)$ is found to be

$$
\frac{d n(x)}{d x} \simeq C \exp [L \cdot F(x)]
$$

where

$$
\begin{aligned}
& F(x)=x \ln \left[(1+a)\left(1+a^{-1} G(x)\right)\right]+(1-x) \ln \left[(1+b G(x))\left(1+b^{-1}\right)\right], \\
& G(x)=\frac{-x+\rho-a b(1-x-\rho)+\sqrt{\left[(x-\rho)^{2}+a b(1-x-\rho)\right]^{2}+4 a b \rho(1-\rho)}}{2 b(1-\rho)} .
\end{aligned}
$$

The constant $C$ in (4.34) is determined by the normalization condition,

$$
\int_{0}^{1} \frac{d n(x)}{d x} d x=1-\tilde{\alpha}-\tilde{\beta} .
$$


It turns out that the function $F(x)$ takes the maximum value at $x_{0}=(1-\tilde{\alpha}-\rho) /(1-\tilde{\alpha}-\tilde{\beta})$. If we expand $F(x)$ around $x=x_{0}$ to the second order and approximate (4.34) by a Gaussian, we find

$$
\frac{d n(x)}{d x} \simeq \sqrt{\frac{L}{2 \pi[\rho(\tilde{\alpha}-\tilde{\beta})+\tilde{\beta}(1-\tilde{\alpha})]}}(1-\tilde{\alpha}-\tilde{\beta})^{2} \exp \left(-\frac{L(1-\tilde{\alpha}-\tilde{\beta})^{2}\left(x-x_{0}\right)^{2}}{2[\rho(\tilde{\alpha}-\tilde{\beta})+\tilde{\beta}(1-\tilde{\alpha})]}\right) .
$$

This is essentially the same expression as that for the totally asymmetric case [15]. But it should be remarked that (4.37) is only a consequence of the above approximation; the correct formula is given by (4.34) with (4.35a) and (4.35b).

We thus obtain the phase diagram shown in Fig. 1. As we have seen, the phase $\mathbf{A}$ (resp. $\mathbf{B})$ for the current subdivides into three phases $\mathbf{A}_{1}, \mathbf{A}_{2}$ and $\mathbf{A}_{3}$ (resp. $\mathbf{B}_{1}, \mathbf{B}_{2}$ and $\mathbf{B}_{3}$ ). This phase diagram has a richer structure than that for the totally asymmetric case [15] for which the phase $\mathbf{A}$ (resp. B) for the current subdivides into only two phases and the phases $\mathbf{A}_{3}$ and $\mathbf{B}_{3}$ are not observed. The situation is analogous to that for the ASEP with open boundaries [18, [19].

\section{Concluding Remarks}

In this article, we have considered the partially asymmetric simple exclusion process on a ring with one defect particle. We have computed the current of the ordinary particles, the speed of the defect particle and the density profile of the ordinary particles seen from the defect particle. The main result of this article is the identification of the phase diagram for the correlation length shown in Fig. 1. It turns out that the phase diagram has a richer structure than that for the totally asymmetric case.

There are several possible applications and generalizations of the analysis in this article. First there are several models for which the partially asymmetric case has not been solved. For instance we can generalize the analysis in [9] for the totally asymmetric case to the partially asymmetric case. Second, we didn't study the case where $q \geq 1$ in this article since our main interest was the phase structure of the model and it is obvious that there is a phase transition at $q=1$. But it would be interesting to apply the similar analysis to this case and compute the physical quantities exactly. In addition, if we consider the limit $q \rightarrow 1$ carefully, the crossover behavior should be observed since this limit is associated with the change of the universality of the model from KPZ universality class to EW universality class [14]. Here we only remark that the corresponding ASEP with open boundaries have been considered in [20] for $q=1$ case and in [21] for $q>1$ case. Lastly it would be also interesting to generalize our analysis to the multi-species models 27 31. Compared to the ASEP, much less is known about these models. Several investigations are now in progress [32]. The results about these will be reported elsewhere. 


\section{Acknowledgment}

The author would like to thank P. Deift, E. R. Speer for fruitful discussions and comments. He also thanks the continuous encouragement of M. Wadati. The author is a Research Fellow of the Japan Society for the Promotion of Science.

\section{Appendix A Density Fluctuation for the Partially ASEP with Open Boundaries}

In the main text of this article, we are mainly interested in the system with a fixed number of particles. The physical quantities are computed for a given value of the density $\rho$. When we consider the ASEP with open boundaries, however, the situation becomes different. The system is connected to particle reservoirs at boundaries; the total number of particles can fluctuate. As pointed out in the main text, the stationary state of the partially ASEP with open boundaries is obtained by setting $\xi=1$ in (2.11). Moreover the probability that the partially ASEP with open boundaries has $N$ particles is given by $Z_{L, N} / Z_{L}(\xi=1)$. By using the asymptotic expressions of $Z_{L}(\xi=1)$ in 18 and those of $Z_{L, N}^{(f)}$ with $f=0, a, b, d$ in (3.17)-(3.20), it is possible to obtain the asymptotic behavior of the probability measure of the particle density when $L \rightarrow \infty$. In particular, we can compute the average and the variance of the density for the partially ASEP with open boundaries. In this appendix, we do not explain even the basic properties of the model and use some terminologies without definition. See [18, 19, 23, 33 35] for instance.

First we study how the asymptotic expression of $Z_{L, N}$ in (3.17)-(3.20) looks like as a function of $0<\rho<1$. As an example we consider $Z_{L, N}^{(a)}$. Let us rewrite (3.17) as

$$
Z_{L, N}^{(a)} \simeq \frac{\left(a^{-1} \xi_{0} ; q\right)_{\infty}}{\sqrt{2 \pi \rho(1-\rho)}\left(b \xi_{0} ; q\right)_{\infty}} \exp [L \cdot S(\rho)]
$$

with

$$
S(\rho)=-\ln \left[\tilde{\alpha}^{1-\rho}(1-\tilde{\alpha})^{\rho} \rho^{\rho}(1-\rho)^{1-\rho}\right] .
$$

Then it is easy to see

$$
\frac{\partial S}{\partial \rho}=\ln \left[\frac{\tilde{\alpha}(1-\rho)}{(1-\tilde{\alpha}) \rho}\right]
$$

becomes zero at $\rho=\tilde{\alpha}$. In addition, we have

$$
\frac{\partial^{2} S}{\partial \rho^{2}}=-\frac{1}{\rho(1-\rho)} \quad(<0)
$$


for $0<\rho<1$. Hence $Z_{L, N}^{(a)}$ takes a single maximum value at $N=\tilde{\alpha} L$. If we expand $S(\rho)$ around $\rho=\tilde{\alpha}$ and substitute it into (A.1), we find

$$
Z_{L, N}^{(A)} \simeq\left[\frac{1}{\tilde{\alpha}(1-\tilde{\alpha})}\right]^{L} \exp \left[-\frac{L(\rho-\tilde{\alpha})^{2}}{2 \tilde{\alpha}(1-\tilde{\alpha})}\right]
$$

when $\rho \sim \tilde{\alpha}$. The width of the peak if of order $L^{-1 / 2}$. As $L$ goes to infinity, we expect that the probability measure of the density is given by a Dirac measure $\delta_{\tilde{\alpha}}$. Similarly $Z_{L, N}^{(b)}$ takes a maximum value at $\rho=1-\tilde{\beta} ; Z_{L, N}^{(0)}$ takes a maximum value at $\rho=1 / 2$. Now we explain the asymptotic behavior of the probability measure of the density and compute the average and the variance of the density.

- low density phase $\left(\tilde{\alpha}<\frac{1}{2}, \tilde{\beta}>\tilde{\alpha}\right)$

From the results in the main text, we see the following. For small densities which satisfy $\rho<1-\tilde{\alpha}$ and $\rho<\tilde{\beta}$, the asymptotic behavior of $Z_{L, N}$ is determined by $Z_{L, N}^{(a)}$. For larger values of $\rho$, we have $Z_{L, N} \simeq Z_{L, N}^{(0)}$ when $1-\tilde{\alpha}<\rho<\tilde{\beta}$ and $Z_{L, N} \simeq Z_{L, N}^{(b)}$ when $\rho>1-\tilde{\alpha}$. As $L$ goes to infinity, however, the main contribution comes from $Z_{L, N}^{(a)}$ in this phase.

By using (A.5), we compute the average and the variance of the density to get

$$
\begin{aligned}
\langle\rho\rangle & =\tilde{\alpha} \\
\left\langle(\rho-\langle\rho\rangle)^{2}\right\rangle & \simeq \frac{\tilde{\alpha}(1-\tilde{\alpha})}{L} .
\end{aligned}
$$

The variance of the density goes to zero as $L \rightarrow \infty$.

- high density phase $\left(\tilde{\beta}<\frac{1}{2}, \tilde{\alpha}>\tilde{\beta}\right)$

For large densities which satisfy $\rho>1-\tilde{\alpha}$ and $\rho>\tilde{\beta}$, the asymptotic behavior of $Z_{L, N}$ is determined by $Z_{L, N}^{(a)}$. For smaller values of $\rho$, we have $Z_{L, N} \simeq Z_{L, N}^{(0)}$ when $1-\tilde{\alpha}<\rho<\tilde{\beta}$ and $Z_{L, N} \simeq Z_{L, N}^{(a)}$ when $\rho<1-\tilde{\alpha}$ and $\rho<\tilde{\beta}$. As $L$ goes to infinity, however, the main contribution comes from $Z_{L, N}^{(b)}$ in this phase.

The average and the variance of the density are found to be

$$
\begin{gathered}
\langle\rho\rangle=1-\tilde{\beta} \\
\left\langle(\rho-\langle\rho\rangle)^{2}\right\rangle \simeq \frac{\tilde{\beta}(1-\tilde{\beta})}{L} .
\end{gathered}
$$

- maximal current phase $\left(\tilde{\alpha}>\frac{1}{2}, \tilde{\beta}>\frac{1}{2}\right)$

For small densities which satisfy $\rho>1-\tilde{\alpha}$, we have $Z_{L, N} \simeq Z_{L, N}^{(a)}$; For large densities which satisfy $\rho>\tilde{\beta}$, we have $Z_{L, N} \simeq Z_{L, N}^{(b)}$. In the middle region of densities which 
satisfy $1-\tilde{\alpha}<\rho<\tilde{\beta}$, the asymptotic behavior of $Z_{L, N}$ is determined by $Z_{L, N}^{(0)}$. As $L$ goes to infinity, the main contribution comes from $Z_{L, N}^{(0)}$ in this phase.

The average and the variance of the density are found to be

$$
\begin{gathered}
\langle\rho\rangle=\frac{1}{2}, \\
\left\langle(\rho-\langle\rho\rangle)^{2}\right\rangle \simeq \frac{1}{8 L} .
\end{gathered}
$$

This formula agrees with the one derived in [35] for the special case $q=0, \alpha=\beta=1$.

- coexistence line $\left(\tilde{\alpha}=\tilde{\beta}<\frac{1}{2}\right)$

For small densities which satisfy $\rho<\tilde{\alpha}$, we have $Z_{L, N} \simeq Z_{L, N}^{(a)}$; For large densities which satisfy $\rho>1-\tilde{\alpha}$, we have $Z_{L, N} \simeq Z_{L, N}^{(b)}$. In the middle region of densities which satisfy $\tilde{\alpha}<\rho<1-\tilde{\alpha}$, the asymptotic behavior of $Z_{L, N}$ is determined by $Z_{L, N}^{(d)}$. When we set $\tilde{\alpha}=\tilde{\beta}$ in (3.20), we notice that $Z_{L, N}^{(d)}$ does not depend on $\rho$. This implies that the probability measure of the density does not have a peak. In fact the probability measure $w(\rho) d \rho$ in the thermodynamic limit is

$$
w(\rho)= \begin{cases}\frac{1}{1-2 \tilde{\alpha}} & \tilde{\alpha}<\rho<1-\tilde{\alpha} \\ 0 & \text { otherwise }\end{cases}
$$

Hence we obtain

$$
\begin{aligned}
\langle\rho\rangle & =\frac{1}{2} \\
\left\langle(\rho-\langle\rho\rangle)^{2}\right\rangle & \simeq \frac{(1-2 \tilde{\alpha})^{2}}{12} .
\end{aligned}
$$

Notice that the variance of the density is finite for this case. This is related to the fact that we see a shock on the coexistence line. The position of the shock can be anywhere [11, 33, 36]. This leads to a macroscopic change of the total number of particles or the finite value of the variance of the density. 


\section{References}

[1] T. M. Ligget, Interacting Particle Systems (Springer-Verlag, New York, 1985).

[2] T. M. Ligget, Stochastic Interacting Systems: Contact, Voter, and Exclusion Processes (Springer-Verlag, New York, 1999).

[3] H. Spohn, Large Scale Dynamics of Interacting Particles (Springer-Verlag, New York, 1991).

[4] B. Schmittmann and R. K. P. Zia, Statistical mechanics of driven diffusive systems, in Phase Transitions and Critical Phenomena, Vol 17, C. Domb and J. Lebowitz eds. (Academic, London, 1994).

[5] M. Schreckenberg and D. E. Wolf eds., Traffic and Granular Flow '97 (Springer Singapore, 1998).

[6] M. R. Evans, N. Rajewsky and E. R. Speer, J. Stat. Phys. 95 (1999) 45.

[7] H.-W. Lee, V. Popkov and D. Kim, J. Phys. A 30 (1997) 8497.

[8] T. Halpin-Healy and Y-C. Zhang, Phys. Rep. 254 (1995) 215.

[9] B. Derrida, S. A. Janowsky, J. L. Lebowitz and E. R. Speer, Europhys. Lett. 22 (1993) 651; J. Stat. Phys. 73 (1993) 813.

[10] B. Derrida, J. L. Lebowitz and E. R. Speer, J. Stat. Phys. 89 (1997) 135.

[11] B. Derrida, S. Goldstein, J. L. Lebowitz and E. R. Speer, J. Stat. Phys. 93 (1998) 547.

[12] J. Krug, Phys. Rev. Lett. 67 (1991) 1882.

[13] L. Gwa and H. Spohn, Phys. Rev. A 46 (1992) 844.

[14] D. Kim, Phys. Rev. E 52 (1995) 3512.

[15] K. Mallick, J. Phys. A 29 (1996) 5375.

[16] B. Derrida and M. R. Evans, J. Phys. A 32 (1999) 4833.

[17] F. H. Jafarpour, cond-mat/9908327.

[18] T. Sasamoto, J. Phys. A 32 (1999) 7109.

[19] T. Sasamoto, cond-mat/9910270.

[20] T. Sasamoto, S. Mori and M. Wadati, J. Phys. Soc. Jpn, 65 (1996) 2000. 
[21] R. A. Blythe, M. R. Evans, F. Colaiori and F. H. L. Essler, cond-mat/9910242

[22] V. Hakim and J. P. Nadal, J. Phys. A 16 (1983) L213.

[23] B. Derrida, M. R. Evans, V. Hakim and V. Pasquier, J. Phys. A 26 (1993) 1493.

[24] R. A. Askey and M. E. Ismail, Memoirs Amer. Math. Soc. 300 (1984).

[25] R. A. Askey and J. A. Wilson, Memoirs Amer. Math. Soc. 319 (1985).

[26] G. Gasper and M. Rahman, Basic Hypergeometric Series (Cambridge, Cambridge, 1990).

[27] M. R. Evans, D. P. Foster, C. Godèche, and D. Mukamel, Phys. Rev. Lett. 74 (1995) 208; J. Stat. Phys. 230 (1995) 69.

[28] M. R. Evans, Y. Kafri, H. M. Koduvely, and D. Mukamel, Phys. Rev. Lett. 230 (1998) 425.

[29] P. F. Arndt, T. Heinzel and V. Rittenberg, J. Phys. A 31 (1998) 833; J. Stat. Phys. 90 (1998) 783; J. Phys. A 31 (1998) L45; cond-mat/9809123.

[30] F. C. Alcaraz, S. Dasmahaptra and V. Rittenberg, J. Phys. A 31 (1998) 845.

[31] K. Mallick, S. Mallick and N. Rajewsky, cond-mat/9903248.

[32] N. Rajewsky, T. Sasamoto and E. R. Speer, in preparation.

[33] G. Schütz and E. Domany, J. Stat. Phys. 72 (1993) 277.

[34] B. Derrida, B. Domany and D. Mukamel, J. Stat. Phys. 69 (1992) 667.

[35] B. Derrida and M. R. Evans, J. Physique I 3 (1993) 311.

[36] A. B. Kolomeisky, G. M. Schütz, E. B. Kolomeisky, J. P. Straley, J. Phys. A 31 (1998) 6911. 


\section{Figure Captions}

Fig. 1 : Phase diagram for the current and the correlation length. The phase boundaries for the current are represented by the thick solid lines; those for the correlation length are

represented by the thin solid lines. The dotted lines are not the phase boundaries. They are drawn for convenience. 


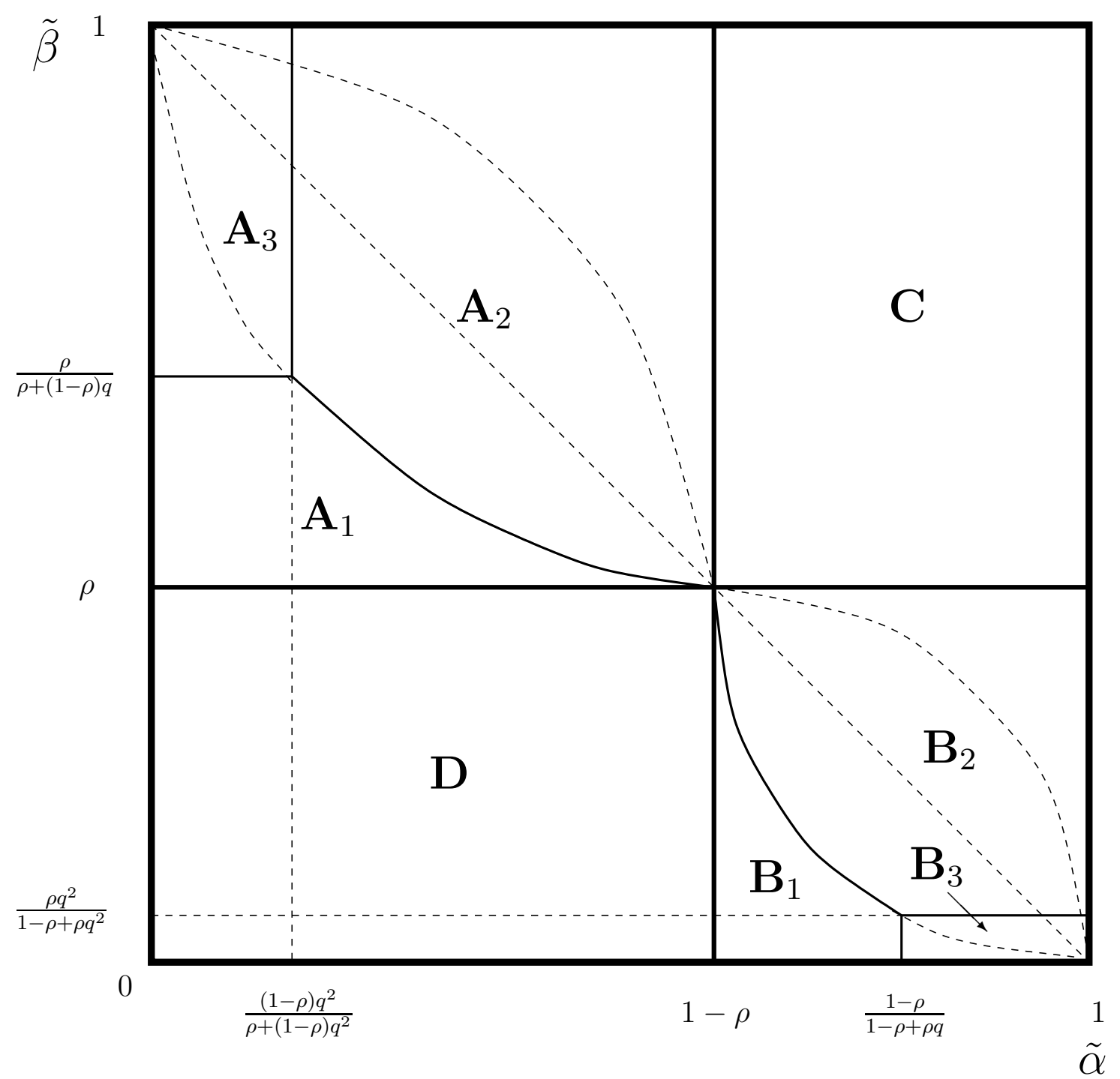

